\title{
Influence of the thickness of monolithic compliant coatings on the skin friction drag
}

\author{
Victor Kulik ${ }^{1, *}$, Andrey Boiko ${ }^{2,3}$, and Inwon $\mathrm{Lee}^{4}$ \\ ${ }^{1}$ ITP SB RAS, Academician Lavrentyev Avenue 1, Novosibirsk, 630090, Russia \\ ${ }^{2}$ ITAM SB RAS, Institutskaya Street 4/1, Novosibirsk, 630000, Russia \\ ${ }^{3}$ University of Tyumen, Volodarskogo Street 6, Tumen, 625003, Russia \\ ${ }^{4}$ GCRC-SOP, Busan daehak-ro 63beon-gil, Geumjeong-gu, Busan, 609-735, Korea
}

\begin{abstract}
In this study, the drag reduction capabilities of compliant coatings made from viscoelastic silicon rubbers are verified experimentally. A series of single-layer compliant coatings of homogeneous materials with constant thicknesses were used. The experiments were performed at different flow velocities in a high-speed water tunnel of Pusan National University. They were accompanied by experimental determination of dynamic viscoelastic properties of the coating materials. Then, for each coating, a dynamic compliance in frequency domain was estimated. Assuming that the coatings interact effectively only with dynamic structures of turbulent boundary layer in a frequency band corresponding to the region about the maximum compliance, promising ranges of flow velocity and coating thickness are outlined. The predicted ranges are compared favourably with the experimental observations in the high-speed water tunnel.
\end{abstract}

\section{Introduction}

Experimental verification of drag reduction capabilities of compliant coatings has long been of great concern. A comprehensive review of such attempts can be found in [1], where most of researches dealt with 'soft' coatings (either a sponge material covered by a thin film or a gel-like substances). It is known that due to the fluid-solid interaction, such soft materials are subject to different instabilities resulting in surface folding and, hence, in drag increase.

The use of more 'stiff' compliant coatings can prevent the surface folds, thereby being advantageous for the sake of drag reduction applications. The surface of the stiff compliant coating tends to remain hydraulically smooth under the action of a flow, as was shown by [2-5]. In a typical water application at flow velocities up to about $20 \mathrm{~m} / \mathrm{s}$, such coatings are characterized by relatively large modulus of elasticity (usually more than $1 \mathrm{MPa}$ ) that prevents the formation of folds and enhances the coating. Although the surface deformation is smaller than the thickness of viscous sublayer, the surface velocity is not negligible.

In the present study, the drag reduction capabilities of single-layer compliant coatings made from viscoelastic silicon rubber are verified experimentally in a high-speed water tunnel. The tests were accompanied by experimental determination of dynamic viscoelastic

* Corresponding author: vkulik@,itp.nsc.ru 
properties of the coating materials to estimate the flow-velocity ranges and the frequency bands for an effective interaction of the coatings with the flow.

\section{Experimental setup}

The experiments were conducted in the high-speed water tunnel of Pusan National University. The test section of the facility is $2 \mathrm{~m}$ long with the cross section being $200 \times 200 \mathrm{~mm} 2$. The water tunnel velocities can be set with the accuracy of $\pm 0.05 \mathrm{~m} / \mathrm{s}$. In addition, each tunnel section is equipped with differential pressure transducers. The turbulence level in the tunnel was $0.6 \%$. The freestream velocity and the pressure gradient were constant with the deviation being less than $2 \%$ throughout measurement time span.

The turbulent boundary layer developed along four walls of the test section. The origin of the streamwise coordinate $x$ was defined at the leading edge of the test section, and no turbulence trip was installed. There were three acrylic windows installed in each of the walls. One of the side windows located downstream was replaced with a floating plate balance unit. The surface of the floating plate balance unit was flush-mounted with the entire side surface of the test section. The upper plate of the floating plate balance unit had $100 \times 50 \mathrm{~mm}^{2}$ rectangular opening for placing a rectangular insert.

The compliant coatings of varying composition and thickness $H$ were mould in the recess of floating plate inserts. The bed surface of the recess was preprocessed with a special primer to provide a strong silicon adhesion. A detailed inspection indicated that the surfaces of the coatings became concave due to a contraction of silicon rubber with time. To flatten the surfaces, the coatings were carefully grinded to keep the surface smooth. Hence, the actual height of the coatings decreased about $2.5 \%$ from the nominal.

Silicone room temperature vulcanizing (RTV) rubbers were used as the compliant coating materials in this study. The RTV 3133 BASE material by Xiameter was polymerized by mixing RTV 3081-F catalyst with the mass composition being $5 \%$ of mixture. The compound was carefully mixed and vacuumed for $10 \sim 15$ minutes in order to get rid of air bubbles which might subsequently form unwanted pores in the sample.

The floating plate inserts with the coatings were installed into an immersed balance. Great care was exercised to maintain the gap between the floating plate and the surrounding surface about $30 \mu \mathrm{m}$ and the level differences less than $5 \mu \mathrm{m}$.

\section{Measurement results}

The main results of frictional force measurements are shown in Fig. 1 for the baseline case (hard insert) and a series of the compliant coatings with varying thicknesses. As seen, the interaction between the coating and the flow depends significantly on the coating thickness. The $4 \mathrm{~mm}$ and $12 \mathrm{~mm}$ thick coatings approach the drag of the hard surface. The $6 \mathrm{~mm}, 8 \mathrm{~mm}$, and $10 \mathrm{~mm}$ thick coatings show similar quite large levels of drag reduction. The changes of the drag with respect to free stream velocity are monotonous.

Viscoelastic properties of a material can be described by the dynamic complex-valued modulus $E(1-i \mu)$, where $E(f)$ and $\mu(f)$ are the real-valued frequency-dependent modulus of elasticity and loss factor, respectively. The experimental method to determine the viscoelastic properties of the coatings is described in detail $[6,7]$. It is based on measuring the vibration amplitude ratio and phase shift between two ends of cylindrical samples. In the present study, the measurements of the viscoelastic properties of the rubber were performed simultaneously with the water-tunnel tests. A simple interpolation of the obtained data of the viscoelastic properties is as follows 


$$
E(f)=\left(3 \cdot 10^{4} \ln (f)+3.67 \cdot 10^{5}\right) \cdot[1-\lambda(0.044 \ln (f)-0.1163)], E_{0}=0.42 \mathrm{MPa} .
$$

It is reasonable to assume that one of the necessary conditions for skin friction drag modification is the maximum interaction of the coating with the flow, the condition does not necessarily differentiate drag reduction or drag increase.

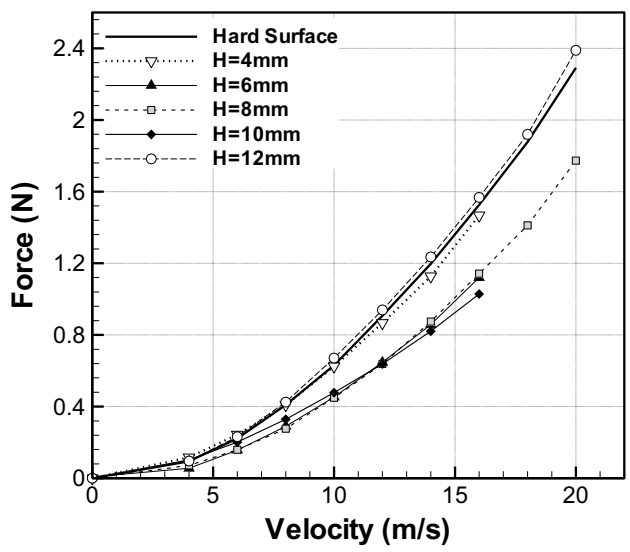

Fig. 1. Friction force for the hard surface and the series of RTV-3133 compliant coatings vs. stream velocity.

The dynamic compliance, i.e. the ratio of dynamic deformation to the applied dynamic mechanical stress (e.g., due to an external periodic forcing), needs to be maximised in order to get the maximum interaction between the surface oscillation and the turbulent pressure fluctuations. It is assumed usually that the normal compliance makes the main contribution to the fluid-coating interaction. The normal compliance $C_{n}$ scaled with the ratio of the static compliance of a long narrow rod of height $H$ with the static modulus of elasticity $E_{0}$ was computed using the measured dynamic properties by the approach described in [2-5] for an infinite plane layer of homogeneous compliant material of height $H$, density $\rho$, Poisson's ratio $\sigma$, modulus of elasticity $E$ and loss tangent $\mu$. It has been shown [2-5] that if the interaction occurs under the action of pressure wave with wavelength $\lambda$ traveling at a speed $V$, the components of the compliance depend only on combinations $\lambda / H$ and $V / C_{t}$, where $C_{t}$ is the propagation velocities of a transverse shear wave travelling in an infinite space without viscous losses $(\mu=0)$ and $f=V / \lambda$ so that $C_{t}^{2}=E(f) /[2 \rho(1+\sigma)]$.

Let us assume that the velocity $V$ of pressure wave induced by a vortical structure in the boundary layer flow is about $0.8 U$, where $U$ is the free-stream flow velocity. This is the upper bound of typical convection velocities discussed by [8], which corresponds to large vortices. If the coating interacts with the smaller-scale relatively high-frequency inner layer structures as assumed, e.g., by [9], $V$ needs to be about $0.69 U$ according to [10].

The modulus $\left|C_{n}\right|$ for the RTV-3133 coatings of different thickness $H$ computed using the empirical formulas are plotted against frequency $f$ and flow velocity $U$ in Fig. 2 . At relatively high velocities (more than about $20 \mathrm{~m} / \mathrm{s}$ ) the compliance is characterized by the presence of a large amplitude ridge stretched parallel to the velocity axis. The maximum compliance occurs at this 'primary' ridge. The position of $\left|C_{n}\right|$ maximum shifts to lower frequencies, as $H$ grows. At velocities about $16 \sim 17 \mathrm{~m} / \mathrm{s}$ the ridge is divided into a pair of 'secondary' ridges with diminishing amplitudes. The location of the secondary ridges is essentially independent of $H$, i.e. it is determined mainly by the viscoelastic properties of material.

Therefore, two scenarios of interaction of the coating with coherent structures of turbulent boundary layer can be proposed: (i) an interaction in a relatively narrow 
frequency range of the 'primary' ridge, which depends on the coating thickness; (ii) a broadband in frequency, but less intensive interaction at lower flow velocities corresponding to the position of the 'secondary' ridges and their downhills. To make a compliant coating durable, its modulus of elasticity should be large. However, as seen, this leads to higher velocities for the efficient interaction at the position of the 'primary' ridge of compliance. A way to overcome this shortcoming is to operate at velocities corresponding to the 'secondary' ridges. Despite the compliance is several times smaller at the 'secondary' ridges than the peak value of the 'primary' ridge, the frequency range of interaction is much wider and the phase variations are much smoother that can lead to wider frequency range of the phase lags favourable for the Reynolds stress reduction.
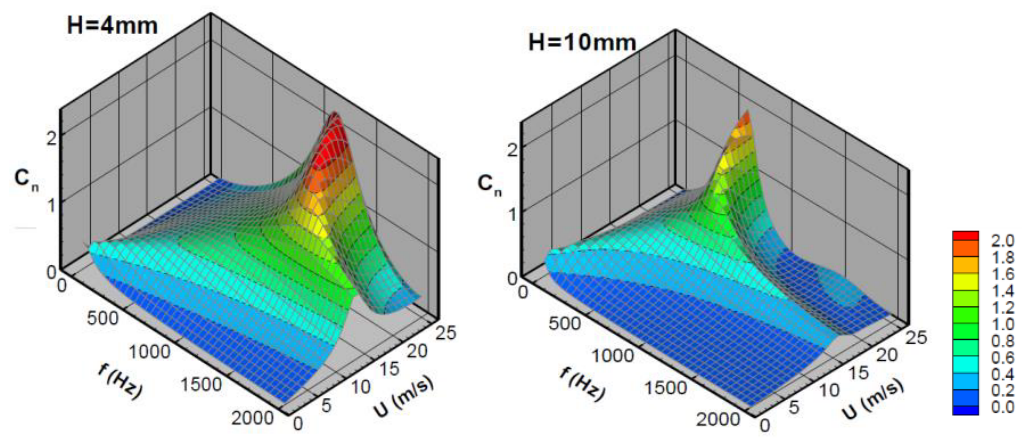

Fig. 2. Computed modulus of normal dynamic compliance of the coatings.

\section{Conclusions}

It was shown that the efficiency of fluid-coating interaction depends on the thickness of coatings in a special way that supports the proposed simplified approach for the prediction of efficiency of the interaction based on the scenario associated with the lower velocity ridges in the compliance charts.

The experiments were financially supported by the National Research Foundation of Korea grants funded by the Ministry of Science, ICT \& Future Planning through GCRC-SOP (No. 2011-0030013) and by the Ministry of Education (No. 2015R1D1A1A01059973). The numerical modelling and data analysis were financially supported by the Russian Science Foundation Grant No. 17-11-01156.

\section{References}

1. M. Gad-el-Hak, Appl. Mech. Rev. 49, 147 (1996)

2. V.M. Kulik, J. Appl. Mech. Tech. Phys. 48, 221 (2007)

3. V.M. Kulik, Thermophys. Aeromech. 16, 43 (2009)

4. V.M. Kulik, Int. J. Heat Fluid Flow 33, 232 (2012)

5. V.M. Kulik, Thermophys. Aeromech. 23, 505 (2016)

6. V.M. Kulik, B.N. Semenov, A.V. Boiko, B.M. Seoudi, H.H. Chun, I. Lee, Exp. Mech. 49, 417 (2009)

7. A.V. Boiko, V.M. Kulik, B.M. Seoudi, H.H. Chun, I. Lee, Int. J. Solids Struct. 47, 374 (2010)

8. B.J. Cantwell, Annu. Rev. Fluid Mech. 13, 457 (1981) 
9. B.N. Semenov, Thermophys. Aeromech. 16, 219 (2009)

10. S.J. Russell, Proc. Int. Symp. Seawater Drag Reduction (Newport, RI, U.S.A., 1998) 